\title{
Deep neck space infection in the paediatric age group
}

\author{
Santosh Kumar Swain, Ranita Sahana \\ Department of Otorhinolaryngology, IMS and SUM Hospital, Siksha "O" Anusandhan University, K8, Kalinganagar, \\ Bhubaneswar, Odisha, India
}

\section{ABSTRACT}

Deep neck space infection (DNSI) is a rare clinical entity in the paediatric age group. However, it can cause significant morbidity and mortality in children. It has insidious presentations but can lead to a fatal and rapid course, which warrants careful observation by the clinician. Common causes of DNSIs are odontogenic and pharyngotonsillar infections. Children with DNSI may present with fever, neck swelling, and breathing difficulty. The fatal complications of DNSI in children are airway obstruction, mediastinitis, internal jugular vein thrombosis, pneumonia, and meningitis. The diagnosis of the DNSI is often challenging to the paediatrician or otolaryngologist. The three important things for successful treatment of the DNSI are antibiotic therapy, surgical drainage, and protection of the airway. Because of the complex anatomy of the neck, the airway management and surgical treatment may be challenging. The aim of this review article is to describe details of aetiopathology, clinical presentation, investigations, and current treatment.

KEY WORDS:

paediatric age, parapharyngeal abscess, peritonsillar abscess, deep neck space infection.

\section{INTRODUCTION}

Deep neck space infection (DNSI) is the infection of the potential spaces and the fascial planes of the neck, either with cellulitis or abscess formation [1]. DNSI is an uncommon disease of the neck among children but may lead to serious life-threatening situations such as upper airway obstruction, thrombosis of the internal jugular vein, and mediastinitis causing high morbidity and mortality [2]. DNSIs are responsible for significant morbidity in the paediatric age group and increase health care expenditures. As per the location of the DNSI, this disease may be classified as different subtypes such as retropharyngeal, parapharyngeal, and peritonsillar abscess [3]. The diagnosis of DNSI is often delayed in children because of the difficulties in physical examination and poor verbal communication of the children [4]. The com- monest clinical presentations are fever and neck swelling [4]. The exact location of the deep neck space infections is well delineated by imaging such as contrast enhanced computed tomography (CT) scan. The treatment includes incision and drainage along with an appropriate antibiotic as per culture sensitive report. There are very few reports for DNSI in the paediatric age group in medical literature. The aim of this review article is to discuss on the aetiopathology, bacteriology, clinical presentations, complications investigations, current treatment, and preventions for DNSI in children.

\section{METHODS OF LITERATURE SEARCH}

Research articles regarding DNSI in the paediatric age group were searched via a multiple approach. First, we conducted an online search of the PubMed, Scopus,

\section{ADDRESS FOR CORRESPONDENCE:}

Santosh Kumar Swain, Department of Otorhinolaryngology, IMS and SUM Hospital, Siksha "O” Anusandhan

University, K8, Kalinganagar, 751003 Bhubaneswar, Odisha, India, ORCID: 0000-0001-7933-4414,

e-mail: santoshvoltaire@yahoo.co.in 
Google Scholar, and Medline databases with the following words/phrases: deep neck space infections in paediatric age, peritonsillar abscess, parapharyngeal abscess, retropharyngeal abscess, aetiopathology, clinical presentations, complications, investigations, current treatment and preventions of the of deep neck space infections. The abstracts of the published articles were identified by this search method, and other articles were identified manually from the citations. This review article reviews the aetiopathology, clinical presentations, complications, diagnosis, current treatment and prevention of DNSI. This review article presents a baseline from which further prospective trials can be designed, and which may help as a spur for further research in this clinical entity for which few studies are performed.

\section{AETIOPATHOLOGY}

DNSIs are usually bacterial infections arising from the upper aero-digestive tract and affecting the deep neck spaces [5]. Common primary sources of infections for DNSI are tooth, tonsils, salivary glands, and retained foreign bodies (Table 1). DNSI usually occurrs after infections such as dental caries, pharyngitis, tonsillitis, and head and neck injuries. The most common source of infection, especially in developing countries, is from an infected tooth or supporting structures, known as odontogenic infections. Before the advent of the antibiotics, peritonsillar space and tonsillar infections were common sources of infections (in approximately $70 \%$ of cases of DNSI), but currently the most common source is odontogenic [6]. Preceding upper airway infections, dental infections, and congenital lesions such as congenital cysts are common causes for DNSIs [2]. Infections of the ear, nose, and throat may spread to the deep neck spaces by direct continuity or through lymphatic drainage to the lymph nodes [7]. Younger children are prone to developing in-

TABLE 1. Aetiology of the deep neck space infection

\begin{tabular}{|l|c|}
\hline Serial number & Aetiology \\
\hline 1 & Odontogenic \\
\hline 2 & Upper airway infections \\
\hline 3 & Peritonsillitis/peritonsillar abscess \\
\hline 4 & Foreign body \\
\hline 5 & Parotitis \\
\hline 6 & Infected thyroglossal cyst \\
\hline 7 & Skin infections \\
\hline 8 & Sialadenitis \\
\hline 9 & Trauma/injury of the neck \\
\hline 10 & Tuberculosis \\
\hline 11 & Idiopathic \\
\hline 12 & \\
\hline
\end{tabular}

fections in the retropharyngeal space because of the presence of the lymph nodes, which spontaneously regress after four to five years of the age [8]. The regression of these neck nodes reduces the chance of the retropharyngeal abscess as the age increases. The pathogenesis of DNSI in children differs from those in the adult age group. Paediatric DNSI is often caused by tonsillitis, pharyngitis, and cervical lymphadenitis whereas in young adults, DNSI is usually associated with odontogenic origin [9].

\section{BACTERIOLOGY}

DNSIs are usually polymicrobial in nature, which includes aerobes, anaerobes, and microaerophilics. The bacteria isolated from pus culture include group A Streptococcus, Peptostreptococcus species, oropharyngeal anaerobic bacteria, and Staphylococcus aureus [10]. Others are Haemophilus influenzae and Klebsiella pneumoniae in immunocompromised patients [11]. It is not always possible to obtain culture because the paediatric patients need sedation and even require general anaesthesia for drainage of the pus and sent for culture sensitivity. One study demonstrated a high risk for methicillin resistant Staphylococcus aureus (MRSA) in paediatric patients younger than two years and association between the MRSA and mediastinitis [12]. Some studies showed that $S$. pyogenes was the commonest pathogen in DNSIs $[13,14]$. These studies were associated with peritonsillar space as the common site for DNSI and prone for S. pyogenes infection. One study showing, pathogens of the DNSI of the children under the age of 10 years was Staphylococcus aureus and pathogens for DNSI of the children or young adult above the age of 10 years showed also similar patterns [15]. As per another study report, majority of the bacteria seems to be aerobic S. viridans, Beta-haemolytic streptococci, Staphylococcus, Klebsiella pneumoniae, anaerobic Bacteroides and Peptostreptococcus [16].

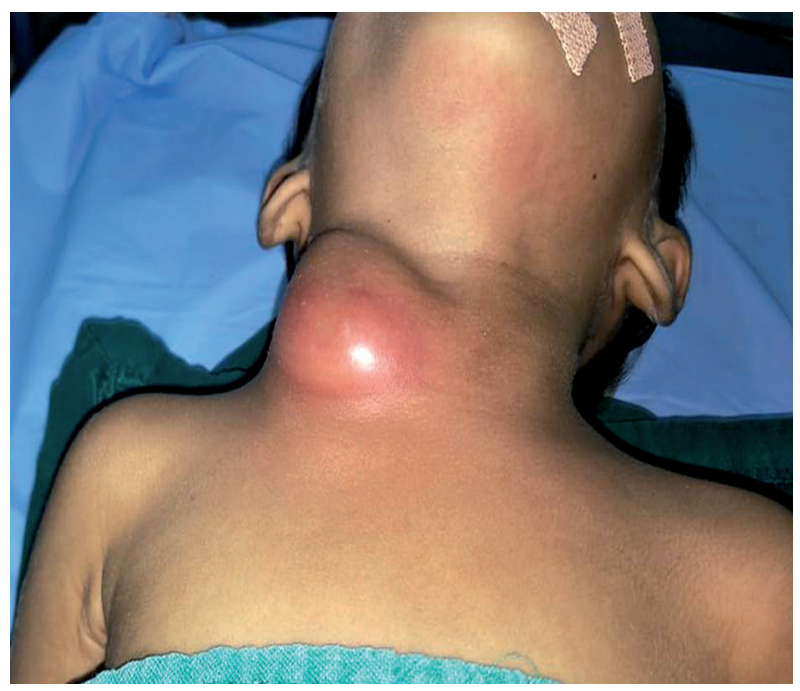

FIGURE 1. A 3-year-old child with DNSI present with swelling of the neck 


\section{ANATOMICAL PERSPECTIVE}

The complex anatomical structures of the neck in paediatric patients make the diagnosis difficult, and exact localisation of the infections in the neck is also challenging. The deep neck spaces extend over the whole length of the neck, which includes the retropharyngeal space, pre-vertebral space, danger space, and the visceral vascular space. The deep neck spaces present above the hyoid bone include submandibular space, parapharyngeal space, peritonsillar space, and masticator space. The only potential neck space situated below the hyoid bone is the anterior visceral space, which is enclosed with middle layer of the deep cervical fascia and contains the oesophagus, trachea, and thyroid gland [17]. DNSI is classified into retropharyngeal, parapharyngeal, parotid, submandibular, and peritonsillar abscess. Accumulation of the pus surrounding the palatine tonsils is called peritonsillar abscess, whereas parapharyngeal abscess is confined medially to the pharynx, posteriorly to the carotid sheath, and laterally to the styloid process and styloid apparatus muscles. The retropharyngeal abscess is usually confined to the pharynx and cervical vertebra and may be confined to the pharynx. Retropharyngeal abscess is confined to the pharynx and cervical vertebra and rarely retropharyngeal. A neck abscess located in more than one is called mixed type DNSI.

\section{CLINICAL PRESENTATIONS}

The clinical presentations of DNSIs are often variable and illustrate difficulty for making the diagnosis on the basis of the symptomatology. The clinical manifestations of the DNSI depend on the basis of the neck spaces involved. The onset of the DNSI is often insidious in onset. The clinical presentations include fever, pain, fatigue, malaise, neck swelling (Fig. 1), odynophagia, trismus, dysphonia, ear pain, and dyspnoea (Table 2). Patients with DNSI may also present with cervical lymphadenopathy, limited neck movements, torticollis, tonsillitis, and displacement of the tonsils. As respiratory distress or stridor is rare; restricted neck movement in a paediatric patient with fever and cervical lymphadenopathy are the sentinel clinical picture for diagnosing DNSI. Although airway symptoms are uncommon, their presence emphasises the morbidity of this infection [8]. The progressive increase of pus formation in the neck space, e.g. retropharyngeal space, is likely to result in narrowing of the upper airway leading to stridor. Children under the age of three years have a high chance of airway obstruction [18]. Hence, DNSI in the young children should be diagnosed quickly and treated promptly. The diagnosis of the DNSI may be delayed because of insidious symptoms and poor verbal communication of paediatric patients, smaller size of the oropharynx, and uncertain abscess presentation in comparison to adults [19]. Clinical suspicions are often
TABLE 2. Clinical presentations in deep neck space infections

\begin{tabular}{|l|c|}
\hline Symptoms & \multicolumn{1}{|c|}{ Signs } \\
\hline Fever & Cervical lymphadenopathy \\
\hline Neck swelling & Restricted neck movement \\
\hline Neck pain & Torticollis \\
\hline Sore throat & Tonsillitis \\
\hline Odynophagia & Tonsillar displacement \\
\hline Dysphagia & Drooling of saliva \\
\hline Respiratory distress & Stridor \\
\hline
\end{tabular}

critical because many deep neck infections are not so evident on inspection or palpation. The male predominance for DNSIs has been seen in many studies, but the underlying explanations are unclear [20,21].

\section{INVESTIGATIONS}

Radiological investigation like X-ray of the neck with lateral view is helpful to evaluate the location for retropharyngeal abscess (Fig. 2). This X-ray acts as screening study; however, it does not differentiate between the retropharyngeal space cellulitis and abscess unless gas is

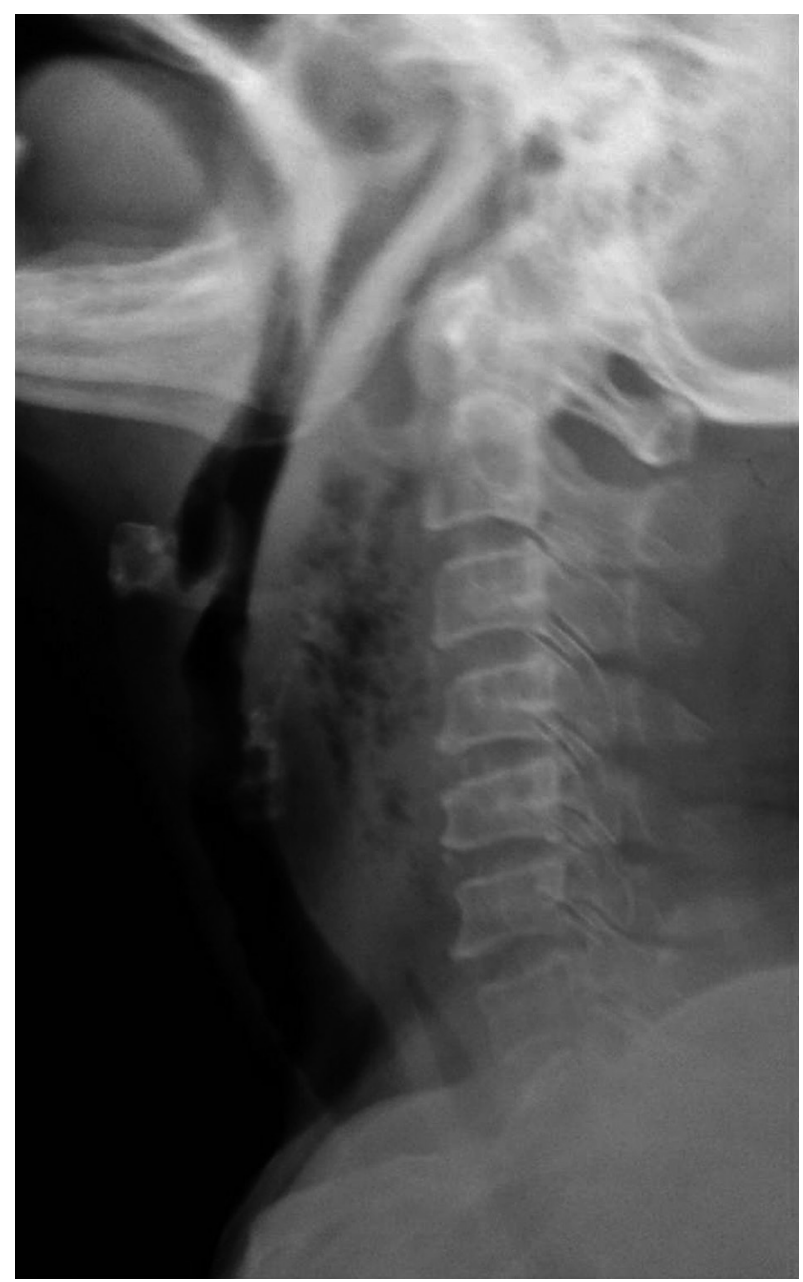

FIGURE 2. X-ray of the neck with lateral view of an 11-year-old boy showing retropharyngeal abscess 
present. X-ray of the neck with lateral view is often taken with adequate neck extension and during the time of the inspiration phase of breathing. Because the proper extension of the neck while taking an X-ray is difficult for children, computed tomography (CT) scan can be recommended when neck abscess is suspected specifically in the retropharyngeal abscess. Ultrasonography is also useful to locate the site of the deep neck space infection. There are few reports supporting for the use of ultrasonography for diagnosis of neck abscess. One study reported that an increase in distance between the internal carotid artery and cervical vertebrae can be used as a criterion, but this study included only five cases of retropharyngeal abscess [22]. CT scan is a widely used modality of the imaging for assessing the exact location of the DNSIs because it is readily available and less expensive [23]. In the case of the recurrence of DNSI, clinicians should think about any underlying bronchogenic cyst. Prompt imaging like CT scan of the neck is required to rule out such congenital lesions and is helpful for adequate treatment. CT scan has the advantages of early and accurate diagnosis, which help for quick planning of treatment and for safe and appropriate drainage [24]. CT scan is an ideal investigation, which is suggested for all patients showing the symptoms and signs of DNSI for better diagnosis. The sensitivity of CT scan for confirming the retropharyngeal abscess is approximately $88-91 \%$ [25]. Blood culture and culture sensitivity of the pus drained from the DNSI are helpful to identify the bacteria and help to provide an appropriate antibiotic for such an infection.

\section{TREATMENT}

Treatments of the DNSI include antibiotic therapy, surgical intervention, and airway management. However, the airway management is always in priority for children with DNSI. A definitive airway must be established if there is any airway compromise. The surgical intervention may be via transoral or transcervical approach. Early diagnosis and prompt treatment are important parts

TABLE 3. Complications found in deep neck space infections

\begin{tabular}{|l|c|}
\hline Serial number & Complications \\
\hline 1 & Airway obstruction \\
\hline 2 & Mediastinitis \\
\hline 3 & Osteomyelitis \\
\hline 4 & Pneumonia \\
\hline 5 & Necrotising fasciitis \\
\hline 6 & Internal jugular vein thrombosis \\
\hline 7 & Sepsis \\
\hline 8 & Disseminated intravascular coagulation (DIC) \\
\hline 9 & Meningitis \\
\hline 10 & Death \\
\hline
\end{tabular}

of prevention of DNSI and its complications. Because of the small anatomical space in the neck, paediatric DNSI requires special skills in terms of the diagnosis and treatment. Traditionally the treatment includes prompt drainage of the abscess followed by the appropriate antibiotics. Conventional treatment of DNSI in the adult age group includes antibiotic treatment and surgical drainage, whereas in the paediatric age group the role of incision or surgical drainage versus medical treatment is still debatable [26]. In DNSI, the antibiotic spectrum should cover both Gram-positive and Gram-negative and both aerobic and anaerobic bacteria. One study suggests amoxicillin clavulanic acid alone or third-generation cephalosporins either alone or in combination with metronidazole as the first-line medication [2]. Some studies documented that antibiotic treatment can useful alone if there is no evidence of a compromised airway. Hence, early diagnosis and prompt treatment are essential for preventing the complications of the DNSI. All paediatric patients with DNSI should be immediately started with an empirical intravenous antibiotic, which should be changed later as per culture and sensitivity report. Before the culture and sensitivity report, the initial desirable antibiotics should be broad spectrum because these infections are polymicrobial in nature. There should be coverage for Gram-negative bacteria in the case of immunocompromised paediatric patients. Clindamycin is the choice of drug as an empirical antibiotic by many clinicians [27], but some clinicians do not recommend it because of the resistance of Streptococci (up to 17\%) to this antibiotic [28]. The antibiotic treatment should be adjusted as per culture and sensitivity report of the pus derived from the DNSI [29]. Because younger children with DNSI have a high chance of airway obstruction, they urgently require diagnosis and prompt treatment [18]. Managing airway obstruction is often challenging in this situation. Children can be prepared by a paediatric anaesthetist for intubation while awake even in an emergent situation. Adequate topicalisation in cooperative children can prevent laryngospasm and acute airway loss, which may necessitate emergency tracheostomy. Then further treatment of surgical drainage is performed. Surgical drainage is considered as primary treatment of this type of young children. However, recently many centres have documented successful non-surgical treatment in a few selected cases. However, the risk factors associated with a transcervical surgical approach for treating paediatric DNSI are unknown. Tracheostomy should be done if airway protection is required [30]. Surgical drainage is the standard method of the treatment in cases of DNSI. The duration of the antibiotic therapy is based on the general condition of the child and wound status, e.g. presence of slough, granulation tissue, or presence of indurations. Mediastinitis with abscess may need thoracic drainage. As was the case in one study, the period of hospital stay for the children receiving abscess drainage was seen to 
be approximately $22 \%$ longer than that of the children receiving medical treatment alone [31]. However, another study showed that the duration of the hospital stay was longer in children taking IV antibiotics in comparison to children with surgical drainage, although the outcomes were not statistically significant [32].

\section{COMPLICATIONS}

Despite widespread use of antibiotics, several lifethreatening and undesirable complications of DNSI are seen in clinical practice (Table 3). Potentially life-threatening complications are seen in $10 \%$ to $20 \%$ of DNSI cases [33]. Infections of the deep neck spaces such as parapharyngeal abscess are common but dangerous in the paediatric age group. They can lead to local, regional, and systemic complications like airway obstruction, mediastinitis, and paraspinal abscess, which may in turn lead to paralysis, jugular vein thrombophlebitis, sepsis, cranial nerve dysfunction, meningitis, cervical osteomyelitis, and death [34]. Other fatal complications in DNSI, although uncommon, include disseminated intra-vascular coagulation, respiratory distress, pleuropulmonary suppuration, and haematogenous dissemination to distant body parts [35]. Upper airway obstruction may require emergency tracheostomy (Fig. 3). DNSI-related mortality depends on the progression of the complications. If necrotising mediastinitis occurs, the mortality rate is approximately $20 \%$ to $40 \%$ [36]. Children with extended neck space infections such as retropharyngeal space abscess and Ludwig's angina present with frequent complications [37].

\section{PREVENTION}

DNSI of paediatric patients is often challenging for otolaryngologists and paediatricians. It should be managed on an emergency basis. Lack of proper nutrition, poor oral hygiene, habit of tobacco chewing, smoking either active or passive, betel nut chewing, habit of eating chocolate by children, and the high prevalence of dental diseases in developing countries lead to vulnerability for DNSI [38]. Hence, prevention of DNSI can be helped by providing health awareness in the community towards raising oral and dental hygiene and encouraging regular dental check-ups for children. It is also vital to provide special attention to children with immunocompromised diseases that may progress into life-threatening complications.

\section{CONCLUSIONS}

Infections of the deep neck spaces often present a true challenge to paediatricians and otolaryngologists. Although the use of broad-spectrum antibiotics in routine clinical practice reduced their incidence, DNSI is still a relevant health problem in the paediatric age group.

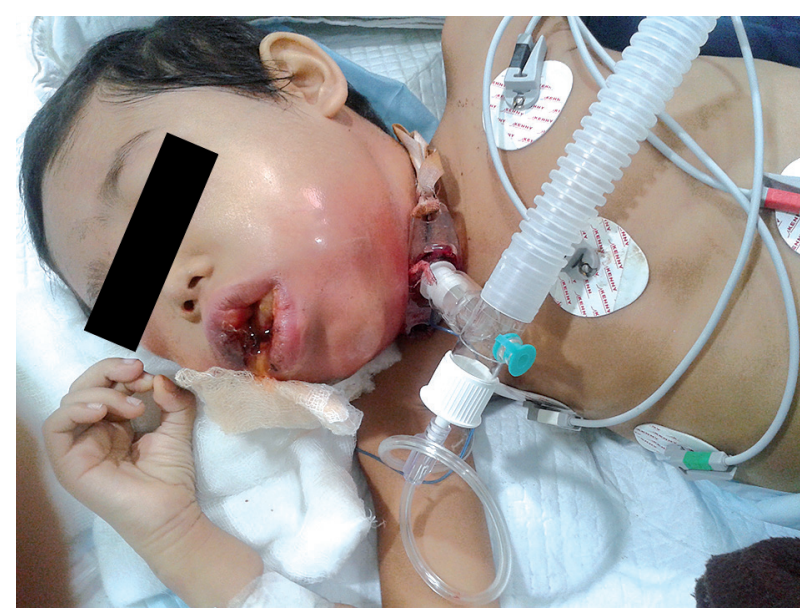

FIGURE 3. A 2-year-old child who underwent tracheostomy for stridor due to DNSI (Ludwig's angina)

Deep neck infections in the paediatric age group can be managed successfully with help of the medical treatment and surgical intervention if diagnosed early. Fatal and emergency situations have been less commonly encountered in recent years because of the post-antibiotic era. However, paediatricians and otolaryngologists must be careful to handle such infections and should not underestimate their potential fatal outcome.

\section{DISCLOSURE}

The authors declare no conflict of interest.

\section{REFERENCES}

1. Wang LF, Kuo WR, Tsai SM, et al. Characterizations of life threatening deep cervical space infections: a review of one hundred ninety six cases. Am J Otolaryngol 2003; 24: 111-117.

2. Huang CM, Huang FL, Chien YL, Chen PY. Deep neck infections in children. J Microbiol Immunol Infect 2017; 50: 627-633.

3. Herzon FS, Martin AD. Medical and surgical treatment of peritonsillar, retropharyngeal, and parapharyngeal abscesses. Curr Infect Dis Rep 2006; 8: 196-202.

4. Novis SJ, Pritchett CV, Thorne MC, et al. Pediatric deep space neck infections in U.S. children, 2000-2009. Int J Pediatr Otorhinolaryngol 2014; 78: 832-836.

5. Durazzo M, Pinto F, Loures M, et al. Deep neck space infections. Rev Ass Med Brasil 1997; 43: 119-126.

6. Parhiscar A, Harel G. Deep neck abscess: a retrospective review of 210 cases. Ann Otolo Rhinol Laryngol 2001; 110: 1051-1054.

7. Coticchia JM, Getnick GS, Yun RD, et al. Age-, site-, and time-specific differences in pediatric deep neck abscesses. Arch Otolaryngol Head Neck Surg 2004; 130: 201-207.

8. Grisaru-Soen G, Komisar O, Aizenstein O, et al. Retropharyngeal and parapharyngeal abscess in children - Epidemiology, clinical features and treatment. Int J of Pediatric Otorhinolaryngol 2010; 74: 1016-1020.

9. Lawrence R, Bateman N. Controversies in the management of deep neck space infection (dnsi) in children: an evidence-based review. Clin Otolaryngol 2017; 42: 156-163.

10. Chang L, Chi H, Chiu NC, et al. Deep neck infections in different age groups of children. J Microbiol Immunol Infect 2010; 43: 47-52. 
11. Lee YQ, Kanagalingam J. Deep neck abscesses: the Singapore experience. Eur Arch Otorhinolaryngol 2011; 268:609-614.

12. Bolton M, Wang W, Hahn A, et al. Predictors for successful treatment of pediatric deep neck infections using antimicrobials alone. Pediatr Infect Dis J 2013; 32: 1034-1036.

13. Raffaldi I, Le Serre D, Garazzino S, et al. Diagnosis and management of deep neck infections in children: the experience of an Italian paediatric centre. J Infect Chemother 2015; 21: 110-113.

14. Cheng J, Elden L. Children with deep space neck infections: our experience with 178 children. Otolaryngol Head Neck Surg 2013; 148: 1037-1042.

15. Daramola OO, Flanagan CE, Maisel RH, et al. Diagnosis and treatment of deep neck space abscesses. Otolaryngol Head Neck Surg 2009; 141: 123-130.

16. Parhiscar A, Har-El G. Deep neck abscess: a retrospective review of 210 cases. Ann Otol Rhinol Laryngol 2001; 110: 1051-1054.

17. Vieira F, Allen SM, Stocks RSM, et al. Deep neck infections. Otolaryngol Clin N Am 2008; 12: 459-483.

18. Swain SK, Sahu MC. Retropharyngeal abscess leading to fatal airway obstruction in a child - a case report. Pediatr Pol 2016; 91: 370-373.

19. Flanary VA, Conley SF. Pediatric deep space neck infections: the Medical College of Wisconsin experience. Int J Pediatr Otorhinolaryngol 1997; 38: 263-271.

20. Wang LF, Tai CF, Kuo WR, et al. Predisposing factors of complicated deep neck infections: 12 -year experience at a single institution. J Otolaryngol Head Neck Surg 2010; 39: 335-341.

21. Ridder GJ, Technau-Ihling K, Sander A, et al. Spectrum and management of deep neck space infections: an 8-year experience of 234 cases. Otolaryngol Neck Surg 2005; 133: 709-714.

22. Chao HC, Chiu CH, Lin SJ, et al. Colour Doppler ultrasonography of retropharyngeal abscess. J Otolaryngol 1999; 28: 138-141.

23. Osborn TM, Assael LA, Bell RB. Deep space neck infection: principles of surgical management. Oral Maxillofac Surg Clin North Am 2008; 20: 353-365.

24. Lee DY, Seok J, Kim YJ, et al. Neck computed tomography in pediatric neck mass as initial evaluation in ED: is it malpractice? Am J Emerg Med 2014; 32: 1237-1240.

25. Ungkanont K, Yellon RF, Weissman JL, et al. Head and neck space infections in infants and children. Otolaryngol Head Neck Surg 1995; $112: 375-382$.

26. Cramer JD, Purkey MR, Smith SS, et al. The impact of delayed surgical drainage of deep neck abscesses in adult and pediatric populations. Laryngoscope 2016; 126: 1753-1760.

27. Lalakea ML, Messner AH. Retropharyngeal abscess management in children: Current practices. Otolaryngol Head Neck Surg 1999; 121: 398-405.

28. Limia A, Jimenez ML, Alarcon T, et al. Five-year analysis of antimicrobial susceptibility of the Streptococcus milleri group. Eur J Clin Microbiol Infect Dis 1999; 18: 440-444.

29. Gidley PW, Ghorayed BY, Stiernberg CW. Contemporary management of deep neck space infections. Otolaryngol Head Neck Surg 1997; 116: 16-22.

30. Swain SK, Sahu MC, Choudhury J, et al. Tracheostomy among pediatric patients: Our experiences at a tertiary care teaching hospital in Eastern India. Pediatr Pol 2018; 93: 312-317.

31. Adil E, Tarshish Y, Roberson D, et al. The public health impact of pediatric deep neck space infections. Otolaryngol Head Neck Surg 2015; 153: 1036-1041.

32. Metin $\mathrm{O}, \mathrm{Oz}$ FN, Tanir G, et al. Deep neck infections in children: experience in a tertiary care center in Turkey. Turk J Pediatr 2014; 56: $272-279$.
33. Boscolo RP, Stellin M, Muzzi E, et al. Deep neck space infections: a study of 365 cases highlighting recommendations for management and treatment. Eur Arch Otorhinolaryngol 2012; 269: 12411249.

34. Huang CM, Huang FL, Chien YL, et al. Deep neck infectionsin children. J Microbiol Immunol Infect 2017; 50: 627-633.

35. Wills PI, Vernon RP. Complications of space infections of the head and neck. Laryngoscope 1981; 91: 1129-1136.

36. Diamantis S, Giannakopoulos H, Chou J, et al. Descending necrotizing mediastinitis as a complication of odontogenic infection. Int J Surg Case Rep 2011; 2: 65-67.

37. Swain SK, Debta P, Sahoo S, et al. An unusual cause of throat pain: a case report. Indian J Public Health Res Dev 2019; 10: 1029-1031.

38. Swain SK, Bhattacharyya B, Sahu MC. An unusual cause of long standing foreign body sensation in throat. Ann Indian Acad Otorhinolaryngol Head Neck Surg 2019; 3: 82. 